\title{
Stroke volume increase to exercise in chronic obstructive pulmonary disease is limited by increased pulmonary artery pressure
}

\author{
S Holverda, ${ }^{1}$ H Rietema, ${ }^{1}$ N Westerhof, ${ }^{1,2}$ J T Marcus, ${ }^{3}$ C T-J Gan,, P E Postmus, ${ }^{1}$ \\ A Vonk-Noordegraaf ${ }^{1}$
}

${ }^{1}$ Department of Pulmonary Diseases, Institute for Cardiovascular Research, VU University Medical Center,

Amsterdam, The Netherlands;

${ }^{2}$ Department of Physiology,

Institute for Cardiovascular

Research, VU University Medical

Center, Amsterdam, The

Netherlands; ${ }^{3}$ Department of

Medical Physics and

Technology, Institute for

Cardiovascular Research, VU

University Medical Center,

Amsterdam, The Netherlands

Correspondence to:

Anton Vonk-Noordegraaf,

Department of Pulmonary

Diseases, VU University Medical

Center Amsterdam, PO Box

7057, 1007 MB Amsterdam,

The Netherlands; a.vonk@

vumc.nl

Accepted 15 April 2008

Published Online First

12 May 2008

\begin{abstract}
Aims: This study was designed to investigate the mechanisms by which the right ventricle is able to increase stroke volume (SV) during exercise in chronic obstructive pulmonary disease (COPD). A second aim was to determine whether resting pulmonary artery pressure (Ppa) is predictive of exercise SV.
\end{abstract}

Methods: 16 COPD patients (GOLD stages II-IV) underwent right heart catheterisation at rest and during exercise. In this group and eight age-matched controls resting and exercise right ventricular SV, end-diastolic volume (RVEDV) and end-systolic volume (RVESV) were assessed by magnetic resonance imaging (MRI). The exercise protocol during both measurements consisted of 3 minutes of cycling in supine position at $40 \%$ of maximal workload.

Results: In all patients mean Ppa increased significantly in response to exercise (21 (8) vs 33 (11) $\mathrm{mm} \mathrm{Hg}$, $\mathrm{p}<0.01)$, whereas pulmonary vascular resistance did not change. In the patient group, RVEDV (129 (42) vs 135 (42) $\mathrm{ml}, \mathrm{p}<0.05$ ) and SV (63 (13) vs 69 (14) $\mathrm{ml}, \mathrm{p}<0.05$ ) increased significantly from rest to exercise, but RVESV and RV ejection fraction remained unaltered. In contrast, in healthy controls SV is augmented (81 (22) vs 101 (28) $\mathrm{ml}, \mathrm{p}<0.05$ ) by both increased RVEDV (123 (33) vs 134 134) $\mathrm{ml}, \mathrm{p}<0.05$ ) and reduced RVESV (37 (9) vs 27 (10) $\mathrm{ml}, \mathrm{p}<0.05)$. Resting mean Ppa was related to SV during exercise $(r=-0.59, p<0.02)$.

Conclusion: As a consequence of unaltered pulmonary vascular resistance to exercise in COPD patients, Ppa increases and SV response to exercise is limited and results from an increased preload only. Ppa at rest predicts exercise SV.

Advanced chronic obstructive pulmonary disease (COPD) is associated with changes in cardiac structure and function owing to increased pulmonary artery pressures. ${ }^{1}$ Before elevated pulmonary artery pressure (Ppa) is apparent at rest, patients with COPD may develop pulmonary hypertension during physical activity. ${ }^{3}$ This abnormal rise in Ppa is explained by the fact that pulmonary vascular resistance (PVR) does not decrease or might even increase during exercise in COPD patients. As a consequence, cardiac output augmentation during exercise is limited and will lead to an increase in Ppa. In contrast, in healthy subjects pulmonary vasodilation, through recruitment and distension of the pulmonary vascular bed, takes place during exercise and pulmonary blood flow increases. Hence, PVR is reduced and the Ppa increase is limited. ${ }^{4}$
Studies on the effects of exercise and the subsequent rise in Ppa on cardiac function have shown that right ventricular end-diastolic volume (RVEDV) increases ${ }^{5}$ and right ventricular ejection fraction (RVEF) fails to augment to exercise in most COPD patients. ${ }^{7-10}$ Right ventricular dilatation and a consequent unaltered RVEF, however, do not necessarily reflect an impaired stroke volume response. ${ }^{11}$ The question remains unanswered to what extent RV pressure changes during exercise affect RV volumes and hence SV response in this patient group.

Therefore, the first objective of this study is to investigate the exercise-induced changes in right ventricular end-diastolic volume and end-systolic volume in relation to stroke volume response in COPD patients and healthy controls; second, whether resting pulmonary arterial pressure is predictive of an abnormal stroke volume response to exercise in this patient group.

\section{METHODS}

\section{Subjects}

This study is part of a larger research project on the development of exercise-induced increases in pulmonary artery pressure in COPD that requires right heart catheterisation and exercise testing in all patients. The VU University medical ethics committee approved the study, and informed consent was obtained from all subjects. Sixteen patients with moderate to severe COPD (GOLD stages II-IV) and increasing symptoms during daily activities despite stable pulmonary function participated in the study. All patients had COPD according to ATS/ERS criteria. ${ }^{12}$ All patients were studied during a stable period of their disease. Patients with a history of cardiovascular disease, in the presence of an abnormal left ventricular function on echocardiography were excluded. A mean $\mathrm{Ppa}>25 \mathrm{~mm} \mathrm{Hg}$ at rest or a mean Ppa $>30 \mathrm{~mm} \mathrm{Hg}$ during exercise confirmed the diagnose of pulmonary hypotension $(\mathrm{PH})$ secondary to COPD. ${ }^{13}$

\section{Study design}

The following three measurements, which are described in more detail below, were performed in all COPD patients within one week on consecutive days: (1) a cardiac MRI scan, which was performed both at rest and during submaximal exercise, (2) a right heart catheterisation both at rest and during submaximal exercise, (3) extensive 
Table 1 Patient demographics and pulmonary function

\begin{tabular}{lll}
\hline & Healthy controls $(\mathbf{n}=\mathbf{8})$ & COPD $(\mathbf{n}=\mathbf{1 6})$ \\
\hline Male/female & $5 / 3$ & $10 / 6$ \\
Age $($ years $)$ & $66(3)$ & $67(9)$ \\
BSA $\left(\mathrm{m}^{2}\right)$ & $1.95(0.16)$ & $1.87(0.16)$ \\
VC $(\%$ predicted $)$ & $118(22)$ & $100(20)$ \\
$\mathrm{FEV}_{1}(\%$ predicted $)$ & $109(16)$ & $51(24)^{* *}$ \\
$\mathrm{FEV}_{1} / \mathrm{NC}(\%)$ & $72(6)$ & $40(17)^{* *}$ \\
TLC $(\%$ predicted) & $103(9)$ & $125(16)^{* *}$ \\
DLCO $(\%$ predicted $)$ & $94(12)$ & $44(17)^{* *}$ \\
\hline
\end{tabular}

Values are mean (SD). BMI, body mass index; COPD, chronic obstructive pulmonary disease; DLCO, carbon monoxide transfer capacity; FEV $_{1}$, forced expiratory volume in one second; TLC, total lung capacity; VC, vital capacity.

${ }^{* *} p<0.01$, versus healthy controls.

lung function testing followed by a maximal cardiopulmonary exercise test. The MRI scan and the right heart catheterisation were performed within 24 hours, and both exercise tests were performed on the same recumbent ergometer (Lode, Groningen, The Netherlands) at identical workload levels. As a control group eight gender-matched and age-matched healthy controls underwent pulmonary function testing and cardiac MRI both at rest and during submaximal exercise. The healthy controls did not undergo right heart catheterisation.

\section{MRI measurements}

The MR images and flow measurements were acquired with a 1.5 Tesla Siemens Sonata whole body system (Siemens Medical Solutions, Erlangen, Germany), equipped with a circularly polarised phased-array body coil. The ECG was recorded with MRI compatible leads, to enable prospective ECG-R wave triggering. The same MRI protocol was used for the resting and exercise measurements, as previously described. ${ }^{14}{ }^{15}$ The MRI exercise protocol consisted of a three-minute period of cycling in supine position on a recumbent bicycle (Lode, Groningen, The Netherlands). For the patient group, work rate was increased in the first minute to $40 \%$ of maximal workload as previously determined during maximal exercise testing. The exercise level for healthy controls was set at $40 \%$ of the predicted maximal workload for gender, age, weight and length. Between exercise measurements a five-minute resting period was included.

To measure RV and LV volumes at end-diastole and endsystole, a stack of short-axis image planes covering the LV and $\mathrm{RV}$ from base to apex was acquired. From the stack of parallel short-axis cine images, quantitative analysis of right ventricular

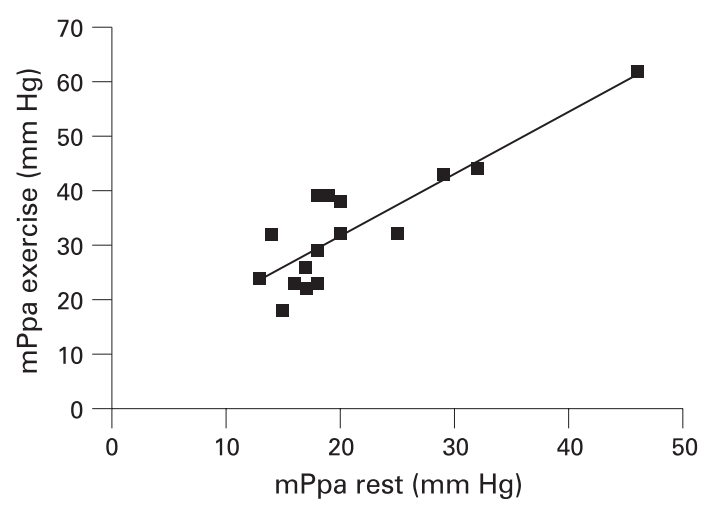

Figure 1 Correlation of resting mean pulmonary artery pressure (mPpa) with exercising $\mathrm{mPpa}$ in 16 patients with chronic obstructive pulmonary disease $(r=0.87, p<0.001)$. volumes and geometry was performed by manual detection of endocardial and epicardial borders on each slice, using the MR Analytical Software System (Medis, Leiden, The Netherlands). Stroke volume (SV) was measured using MR phase-contrast flow quantification. ${ }^{14}$

\section{Right heart catheterisation}

The tests were performed in the intensive care unit with the patient in stable condition breathing room air and continuous monitoring of ECG and systemic blood pressures. The right heart catheterisation was performed with a balloon-tipped, flow-directed 7F Swan-Ganz catheter (131HF7; Baxter Healthcare Corp; Irvine, CA, USA). Pulmonary artery pressures were taken at the end of expiration. Cardiac output was determined with the direct Fick method. Pulmonary vascular resistance was calculated as the ratio of mean pressure to cardiac output. Haemodynamic measurements were obtained at baseline and while cycling. The exercise protocol consisted of a three-minute period of cycling in supine position on a recumbent bicycle (Lode, Groningen, The Netherlands) with the Swan-Ganz catheter in situ. Work rate was increased in the first minute to $40 \%$ of maximal workload as previously determined during maximal exercise testing (as described below) and was identical to exercise MRI measurements.

\section{Lung function and exercise testing}

Pulmonary function was evaluated by standard spirometry, determination of carbon monoxide transfer capacity (DLCO), and measurement of functional residual capacity (FRC) and total lung capacity (TLC) following ERS/ATS guidelines. ${ }^{16-18}$ General characteristics and pulmonary function data are shown in table 1.

Maximal exercise tolerance and peak oxygen uptake $\left(\mathrm{VO}_{2}\right)$ were assessed by a standard, incremental, maximal exercise test on an electronically braked cycle ergometer (Lode, Groningen, The Netherlands). Measurements of $\mathrm{VO}_{2}$ and carbon dioxide output were made breath-by-breath (Vmax229, Sensormedics, Yorba Linda, CA, USA). The exercise protocol consisted of 3 minutes of rest, 3 minutes of unloaded cycling at $60 \mathrm{rpm}$ followed by a progressively increasing work rate to maximum tolerance and 3 minutes of recovery. ${ }^{19}$

\section{Statistics}

Data are presented as mean (SD). The SPSS 12.0 software package was used for statistical analyses and a value of $p<0.05$ was considered significant. A Wilcoxon signed rank test was used to compare between resting and exercise conditions. The Mann-Whitney $U$ test was applied to compare cardiac function between healthy controls and COPD patients.

Table 2 Right heart catheterisation results in COPD patients

\begin{tabular}{lcc}
\hline & Rest & Exercise \\
\hline $\mathrm{sPpa}(\mathrm{mm} \mathrm{Hg})$ & $35(15)$ & $55(18)^{* *}$ \\
$\mathrm{dPpa}(\mathrm{mm} \mathrm{Hg})$ & $12(7)$ & $19(11)^{* *}$ \\
$\mathrm{mPpa}(\mathrm{mm} \mathrm{Hg})$ & $21(8)$ & $33(11)^{* *}$ \\
$\mathrm{PVR}\left(\right.$ dynes $\left.\cdot \mathrm{s}^{-1} \cdot \mathrm{cm}^{-5}\right)$ & $276(170)$ & $280(171)$ \\
$\mathrm{SaO}_{2}(\%)$ & $92(4)$ & $88(6)^{* *}$ \\
$\mathrm{mvSaO}_{2}(\%)$ & $67(7)$ & $51(8)^{* *}$ \\
\hline
\end{tabular}

Values are expressed as mean (SD).

COPD, chronic obstructive pulmonary disease; $\mathrm{dPpa}$, diastolic pulmonary artery pressure; $\mathrm{mPpa}$, mean pulmonary artery pressure; $\mathrm{mvSaO}_{2}$, mixed venous oxygen saturation; PVR, pulmonary vascular resistance; $\mathrm{SaO}_{2}$, arterial oxygen saturation; $\mathrm{sPpa}$, systolic pulmonary artery pressure. ${ }^{* *} \mathrm{p}<0.01$, vs rest. 
Table 3 Cardiac structure and function at rest and during exercise in healthy controls and COPD patients

\begin{tabular}{|c|c|c|c|c|}
\hline & \multicolumn{2}{|c|}{ Healthy controls } & \multicolumn{2}{|c|}{ COPD patients } \\
\hline & Rest & Exercise & Rest & Exercise \\
\hline HR (beats/min) & $71(8)$ & $96(12)^{*}$ & $81(11) \dagger$ & $96(14)^{* *}$ \\
\hline SV (ml/beat) & $81(22)$ & $101(28)^{*}$ & $6313) \dagger$ & $69(14)^{*}+$ \\
\hline CO (1/min) & $5.7(1.5)$ & $9.6(2.6)^{*}$ & $5.1(1.2)$ & $6.7(1.9)^{* *} \dagger$ \\
\hline \multicolumn{5}{|l|}{ Right ventricle } \\
\hline EDV (ml) & $127(35)$ & $136(35)^{*}$ & $129(42)$ & $135(42)^{*}$ \\
\hline ESV (ml) & $46(17)$ & $35(15)^{*}$ & $65(46)$ & $65(48) \dagger$ \\
\hline $\mathrm{EF}(\%)$ & $64(6)$ & $74(8)^{*}$ & $52(13):$ & $54(14):$ \\
\hline \multicolumn{5}{|l|}{ Left ventricle } \\
\hline EDV (ml) & $121(35)$ & $129(36)^{*}$ & $94(16)$ & $98(17) \dagger$ \\
\hline ESV (ml) & $40(15)$ & $28(14)^{*}$ & $31(12)$ & $29(12)$ \\
\hline EF (\%) & $68(6)$ & $79(8)^{*}$ & $67(11)$ & $71(11)^{*}$ \\
\hline
\end{tabular}

Values are expressed as mean (SD). CO, cardiac output; COPD, chronic obstructive pulmonary disease; EDV, end-diastolic volume; $\mathrm{EF}$, ejection fraction; ESV, end-systolic volume; HR, heart rate; SV, stroke volume.

${ }^{*} \mathrm{p}<0.05$, ${ }^{* *} \mathrm{p}<0.01$, rest versus exercise measurements, $\dagger \mathrm{p}<0.05$, versus healthy controls, $\$ \mathrm{p}<0.01$ versus healthy controls.

Pearson correlation analyses were calculated to determine the correlations between haemodynamic and cardiac function data.

\section{RESULTS}

\section{General characteristics}

The results of the right heart catheterisation of the COPD patients both at rest and during submaximal exercise are presented in table 2. Nine out of 16 patients were diagnosed with $\mathrm{PH}$ : four patients showed $\mathrm{PH}$ at rest, in five patients exercise induced an increase in $\mathrm{mPpa}$ above $30 \mathrm{~mm} \mathrm{Hg}$. In all patients Ppa increased significantly in response to exercise, whereas pulmonary vascular resistance did not change. As shown in figure 1 , exercise $\mathrm{mPpa}$ is related to resting $\mathrm{mPpa}$ in COPD patients.

Cardiopulmonary exercise test results from the COPD patients yielded a maximal workload of $36 \%$ (SD 18\%) (percentage of predicted), a peak oxygen uptake of $49 \%$ (SD $12 \%$ ) (percentage of predicted), peak ventilation of $66 \%$ (SD $16 \%$ ) (percentage of predicted), ventilatory equivalent for $\mathrm{CO}_{2}$ at nadir of 48 (SD 15), peak oxygen pulse of 50\% (SD 17\%)

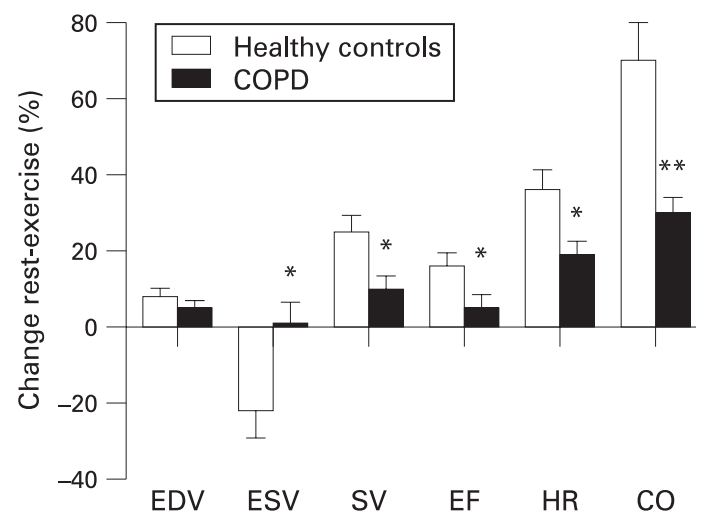

Figure 2 Change in right ventricular structure and function in response to submaximal exercise in both patients with chronic obstructive pulmonary disease (COPD) and healthy controls. Note that although right ventricular end-diastolic volume (EDV) is increased in both groups, in contrast to COPD patients, the healthy controls have the ability to reduce right ventricular end-systolic volume (ESV). This results in an improved stroke volume (SV) and ejection fraction (EF). CO, cardiac output; HR, heart rate. ${ }^{*} p<0.05$, versus healthy controls, ${ }^{* *} p<0.01$, versus healthy controls. (percentage of predicted) and a slope of oxygen uptake relative to workload of 7.2 (SD 2.4).

\section{Cardiac function}

All patients and healthy subjects were able to perform the MRI exercise test at $40 \%$ of their maximal workload. Right and left ventricular characteristics at rest and during exercise of both groups are presented in table 3 . Both heart rate and stroke volume were significantly augmented in exercise. However, the changes were significantly larger in the control group, and, as a consequence, cardiac output increased much more in the control group.

Figure 2 demonstrates the differences in right ventricular response to submaximal exercise between healthy controls and COPD patients. In the patient group, right ventricular enddiastolic volume (RVEDV) and SV increased significantly from rest to exercise, but RV end-systolic volume and RVEF did not. While in the COPD group SV is solely increased because of an increased RVEDV, SV in healthy controls is raised by both an increased RVEDV and a reduced RVESV. Stroke volume at rest and during exercise was significantly smaller in the patient group in comparison with healthy controls.

When the absolute change to exercise (exercise minus resting values) in SV is plotted against the absolute change in RVESV (fig 3), it shows that in three patients RVESV is increased and SV is reduced during exercise. These three patients

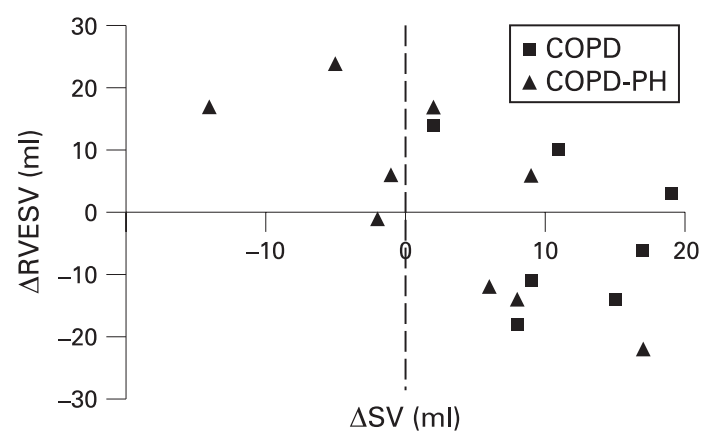

Figure 3 The absolute changes from rest to exercise in stroke volume (SV) plotted against the absolute changes in right ventricular end-systolic volume (RVESV) in patients with chronic obstructive pulmonary disease (COPD). In three patients RVESV is increased and SV is reduced during exercise. These three patients demonstrated increased levels of pulmonary artery pressure at rest. 
demonstrated increased levels of $\mathrm{Ppa}$ at rest. In all COPD patients without $\mathrm{PH}$ at rest or during exercise SV was increased by exercise. Almost half the patients showed an augmentation of both RVESV and SV. As shown in figure 3, there is a significant inverse relation between the absolute change in exercise in both SV and RVESV $(r=-0.56, p<0.03)$.

\section{Relation pulmonary artery pressure and cardiac function}

To investigate the predictive value of $\mathrm{mPpa}$ at rest on right ventricular structure and function during exercise, we assessed the relation between resting $\mathrm{mPpa}$ and SV, RVEDV and RVEF during exercise. Resting $\mathrm{mPpa}$ was inversely related to $\mathrm{SV}$ during exercise $(r=-0.59, \mathrm{p}<0.02)$. Furthermore, mPpa at rest showed a correlation with both RVEDV and RVEF during exercise $(r=0.75, p<0.001$ and $r=-0.80, p<0.001$, respectively). In addition, a high $\mathrm{mPpa}$ during exercise is associated with a large RVESV during exercise.

\section{DISCUSSION}

This is the first cardiovascular MR study to show the mechanism by which right ventriclular stroke volume is augmented during exercise in COPD patients. The results show a limited increase in stroke volume to submaximal exercise, mainly resulting from larger EDV. In healthy controls, stroke volume augmentation to exercise was the result of both an increased right ventricular end-diastolic volume and a reduction in end-systolic volume. In addition, we found that in COPD a high resting $\mathrm{mPpa}$ was predictive for a low stroke volume during exercise.

\section{Pulmonary artery pressure and stroke volume}

Whereas pulmonary artery pressures may be elevated during daily activities in COPD patients, ${ }^{30}$ in general pulmonary hypertension at rest is only mild to moderate. ${ }^{21}$ Although, pulmonary artery pressures are modestly increased at rest, our results together with earlier studies showed that COPD patients have a reduced SV at rest. ${ }^{1}$ However, SV at rest was similar between controls and COPD patients when indexed for body surface area (41 (10) $\mathrm{ml}$ vs 34 (7) $\mathrm{ml}, \mathrm{p}=0.14$, respectively). In addition, exercise leads to a rapid increase in pulmonary artery pressure even in patients with normal resting pressures. This increase in Ppa results from increased PVR as a consequence of hypoxic pulmonary vasoconstriction and a reduced pulmonary capillary bed. ${ }^{21}$ High Ppa levels may furthermore be explained by an increase in pulmonary vascular closure that can be accounted for by volume-induced stretching of alveolar vessels and by increased alveolar pressures as a consequence of dynamic hyperinflation. Hypoxic pulmonary vasoconstriction and remodelling could also lead to pulmonary vascular closure, and hence increased right ventricular afterload. ${ }^{22}$ One of the major findings of our study is that an increased pulmonary artery pressure at rest is related to a low stroke volume during exercise. In patients with idiopathic pulmonary artery hypotension (mPpa at rest $=51(18) \mathrm{mm} \mathrm{Hg}$ ) we have previously shown that, in spite of a small increase in RVEDV, SV was not augmented and LVEDV was decreased to exercise. ${ }^{15}$ Although, SV increased in the COPD group, this increase was modest and absent in three out of four COPD patients with $\mathrm{PH}$ at rest. In contrast to patients with idiopathic $\mathrm{PH}$, increased $\mathrm{RV}$ preload during exercise did not affect left ventricular end-diastolic volume in the present patient group. The results suggest that cardiac performance during exercise in COPD patients can be staged between healthy controls and patients with advanced types of pulmonary arterial hypertension.

\section{Right ventricular function}

In the literature, there are few data concerning the effects of exercise on right ventricular function in both healthy subjects and COPD patients. An earlier study in young healthy adults has revealed that an increase in stroke volume during submaximal exercise in upright position is mainly caused by an increased preload of both the right and left ventricle. ${ }^{23}$ During supine maximal exercise, however, it was shown that in healthy controls SV was enhanced owing to a decrease in RVESV. ${ }^{24}{ }^{25}$ The latter authors conclude that the enhanced right ventricular SV during progressive supine exercise seems more related to increased contractility than to the Frank-Starling mechanism. Mahler and co-workers used a combined haemodynamic and radionuclide approach to study RV performance during upright exercise in 12 COPD patients. ${ }^{7}$ Their data showed that RVEF remained unchanged during exercise, despite considerable changes in RVEDV and RVESV in individual patients. This finding is in agreement with our study. However, in Mahler's study changes in right ventricle volumes during exercise were not related to stroke volume. In a group of 25 stable COPD patients, Biernacki and co-workers ${ }^{6}$ analysed the slope of the right ventricular end-systolic pressurevolume relation at rest and during exercise and concluded from their findings that despite the presence of $\mathrm{PH}$ right ventricular contractility remained relatively normal. In addition, in agreement with the present study, left ventricular function was shown to be preserved at rest ${ }^{1}$ and during exercise, $^{8}$ whereas RVEF and $\mathrm{mPpa}$ were found to be related in COPD patients. ${ }^{26}$ What is new in our study is that we sought to find the relation between changes in SV and right ventricular volumes in order to gain a better understanding of the mechanism of impaired stroke volume response to exercise in COPD patients.

Our data showed that the elderly age-matched controls increase their stroke volume during exercise in supine position by increasing RVEDV and reducing RVESV. Although an increase in RVEDV was observed in the COPD patients, these patients failed to reduce RVESV. Consequently, stroke volume response was augmented whereas RVEF remained unaltered during exercise in these patients.

Based on these findings we conclude that both controls and COPD patients use the Frank Starling mechanism to augment $\mathrm{SV}$ in response to exercise. In controls RV contractility increased, Ppa remained unaltered and RVESV decreased. In COPD patients RV contractility was also increased, which would normally decrease RVESV, but the simultaneous increase in Ppa impedes ejection, resulting in an unchanged RVESV. The increase in Ppa is in part the result of the PVR that remains constant during exercise. Therefore, in contrast to controls the SV change to exercise results from an increase in preload only. To generate these high pressures and to maintain SV during exercise, the work of the right ventricle is disproportionally increased in COPD patients in comparison with healthy controls. Note that as a consequence of the study design (that is, different exercise levels between patients and healthy controls) we were able to show differences in the mechanism by which a maximal SV is induced during exercise; however, from our results we cannot conclude whether this RV response is normal or abnormal in the patient group. 


\section{Study limitations}

Simultaneous pressure measurements and cardiac MRI could not be performed in our institute. Both measurements were therefore performed within 24 hours. A maximal supine exercise test appeared to be difficult to perform during MRI and too uncomfortable for the patients during right heart catheterisation. Therefore, a submaximal exercise level of $40 \%$ of previously determined maximal exercise level was used to assure a maximal stroke volume response. ${ }^{27}$ The same exercise level and bicycle ergometer was used during both tests.

\section{Conclusion}

In conclusion, in COPD patients SV increase is limited and results from an increase in end-diastolic volume only, and not from a reduced end-systolic volume. Impaired right ventricular contractility is related to pulmonary artery pressure.

Funding: None.

Competing interests: None.

\section{REFERENCES}

1. Vonk-Noordegraaf A, Marcus JT, Holverda S, et al. Early changes of cardiac structure and function in COPD patients with mild hypoxemia. Chest 2005; 127:1898-903.

2. Burrows B, Kettel LJ, Niden AH, et al. Patterns of cardiovascular dysfunction in chronic obstructive lung disease. N Engl J Med 1972;286:912-8.

3. Raeside DA, Brown A, Patel KR, et al. Ambulatory pulmonary artery pressure monitoring during sleep and exercise in normal individuals and patients with COPD. Thorax 2002; 57:1050-3.

4. Lonsdorfer-Wolf E, Richard R, Doutreleau S, et al. Pulmonary hemodynamics during a strenuous intermittent exercise in healthy subjects. Med Sci Sports Exerc 2003:35:1866-74.

5. Mahler DA, Brent BN, Loke J, et al. Right ventricular performance and central circulatory hemodynamics during upright exercise in patients with chronic obstructive pulmonary disease. Am Rev Respir Dis 1984;130:722-9.

6. Biernacki W, Flenley DC, Muir AL, et al. Pulmonary hypertension and right ventricular function in patients with COPD. Chest 1988;94:1169-75.

7. Mahler DA, Brent BN, Loke J, et al. Right ventricular performance and central circulatory hemodynamics during upright exercise in patients with chronic obstructive pulmonary disease. Am Rev Respir Dis 1984;130:722-9.

8. Matthay RA, Berger HJ, Davies RA, et al. Right and left ventricular exercise performance in chronic obstructive pulmonary disease: radionuclide assessment. Ann Intern Med 1980;93:234-9.
9. Morrison DA, Adcock K, Collins CM, et al. Right ventricular dysfunction and the exercise limitation of chronic obstructive pulmonary disease. J Am Coll Cardiol 1987:9:1219-29.

10. Oliver RM, Fleming JS, Waller DG. Right ventricular function at rest and during exercise in chronic obstructive pulmonary disease. Comparison of two radionuclide techniques. Chest 1993;103:74-80.

11. Weitzenblum $\mathbf{E}$, Chaouat $\mathrm{A}$. Right ventricular function in COPD: can it be assessed reliably by the measurement of right ventricular ejection fraction? Chest 1998:113:567-9.

12. Celli BR, MacNee W. Standards for the diagnosis and treatment of patients with COPD: a summary of the ATS/ERS position paper. Eur Respir J 2004;23:932-46.

13. Barst RJ, McGoon M, Torbicki A, et al. Diagnosis and differential assessment of pulmonary arterial hypertension. J Am Coll Cardiol 2004;43:40S-47S

14. Marcus JT, Vonk-Noordegraaf A, De Vries PM, et al. MRI evaluation of right ventricular pressure overload in chronic obstructive pulmonary disease. J Magn Reson Imaging 1998:8:999-1005.

15. Holverda S, Gan CT, Marcus JT, et al. Impaired stroke volume response to exercise in pulmonary arterial hypertension. J Am Coll Cardiol 2006;47:1732-3.

16. Miller MR, Hankinson J, Brusasco V, et al. Standardisation of spirometry. Eur Respir J 2005;26:319-38.

17. MacIntyre N, Crapo RO, Viegi G, et al. Standardisation of the single-breath determination of carbon monoxide uptake in the lung. Eur Respir J 2005;26:720-35

18. Wanger J, Clausen JL, Coates A, et al. Standardisation of the measurement of lung volumes. Eur Respir J 2005;26:511-22.

19. Wasserman K, Hansen J, Sue DY, et al. Principles of the exercise testing and interpretation. 3rd ed. Baltimore, MD: Lippincott Williams \& Wilkins, 1999.

20. Christensen CC, Ryg MS, Edvardsen A, et al. Relationship between exercise desaturation and pulmonary haemodynamics in COPD patients. Eur Respir $J$ 2004;24:580-6.

21. Barbera JA, Peinado VI, Santos S. Pulmonary hypertension in chronic obstructive pulmonary disease. Eur Respir J 2003;21:892-905.

22. Naeije R, MacNee W. Pulmonary circulation. In: Calverley P, MacNee W, Pride N, et al, eds. Chronic obstructive pulmonary disease. 2nd ed. London: Arnold Health Sciences, 2003:228-42.

23. Mahler DA, Matthay RA, Snyder PE, et al. Volumetric responses of right and left ventricles during upright exercise in normal subjects. J App/ Physiol 1985;58:1818-22.

24. Manyari DE, Kostuk WJ. Left and right ventricular function at rest and during bicycle exercise in the supine and sitting positions in normal subjects and patients with coronary artery disease. Assessment by radionuclide ventriculography. Am J Cardiol 1983; $\mathbf{5 1 : 3 6 - 4 2 . ~}$

25. Mols $\mathbf{P}$, Huynh $\mathrm{CH}$, Naeije $\mathrm{N}$, et al. Volumetric response of right ventricle during progressive supine exercise in men. Am J Physiol 1991;261:H751-4.

26. Brent BN, Berger HJ, Matthay RA, et al. Physiologic correlates of right ventricular ejection fraction in chronic obstructive pulmonary disease: a combined radionuclide and hemodynamic study. Am J Cardiol 1982;50:255-62.

27. Astrand PO, Cuddy TE, Saltin B, et al. Cardiac output during submaximal and maximal work. J Appl Physiol 1964;19:268-74. 


\section{HEART}

\section{Stroke volume increase to exercise in chronic obstructive pulmonary disease is limited by increased pulmonary artery pressure}

S Holverda, H Rietema, N Westerhof, et al.

Heart 2009 95: 137-141 originally published online May 12, 2008 doi: $10.1136 /$ hrt.2007.138172

Updated information and services can be found at:

http://heart.bmj.com/content/95/2/137.full.html

\section{These include:}

References This article cites 25 articles, 16 of which can be accessed free at: http://heart.bmj.com/content/95/2/137.full.html\#ref-list-1

Article cited in:

http://heart.bmj.com/content/95/2/137.full.html\#related-urls

Email alerting Receive free email alerts when new articles cite this article. Sign up in service the box at the top right corner of the online article.

Topic Articles on similar topics can be found in the following collections

Collections

Clinical diagnostic tests (20445 articles)

Notes

To request permissions go to:

http://group.bmj.com/group/rights-licensing/permissions

To order reprints go to:

http://journals.bmj.com/cgi/reprintform

To subscribe to BMJ go to:

http://group.bmj.com/subscribe/ 